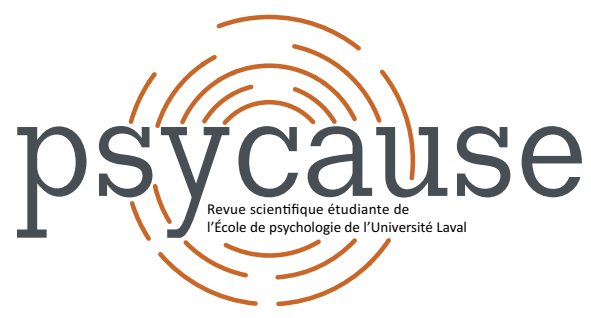

\title{
PSYCAUSE
}

Revue scientifique étudiante de l'École de psychologie de l'Université Laval

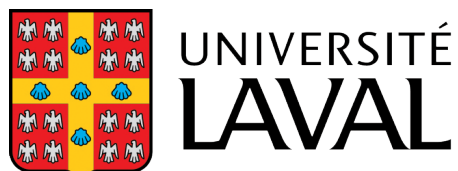

Faculté des sciences sociales École de psychologie

\section{ATTACHEMENT, PERSONNALITÉ ET VIOLENCE CONJUGALE PSYCHOLOGIQUE CHEZ DES FEMMES EN PSYCHOTHÉRAPIE}

Gabrielle CLOUTIER ${ }^{1, \star}$, Marie-Chloé NOLIN ${ }^{1}$, Laurence CÔTÉ ${ }^{1}$, Stéphane SABOURIN ${ }^{1}$ et Vincent BELLEMARE ${ }^{1}$

${ }^{1}$ École de psychologie, Université Laval

*gabrielle.cloutier.4@ulaval.ca

\section{Pour citer l'article}

Cloutier, G., Nolin, M.-C., Côté, L., Sabourin, S., \& Bellemare, V. (2019). Attachement, personnalité et violence conjugale psychologique chez des femmes en psychothérapie. Psycause: Revue scientifique étudiante de l'École de psychologie de l'Université Laval, 9(2), 5-6. 


\title{
ATTACHEMENT, PERSONNALITÉ ET VIOLENCE CONJUGALE PSYCHOLOGIQUE CHEZ DES FEMMES EN PSYCHOTHÉRAPIE
}

\author{
Gabrielle CLOUTIER ${ }^{1, \star}$, Marie-Chloé NOLIN ${ }^{1}$ Laurence CÔTÉ ${ }^{1}$, Stéphane SABOURIN \& Vincent BELLEMARE ${ }^{1}$ \\ 1 École de psychologie, Université Laval \\ *gabrielle.cloutier.4@ulaval.ca
}

\section{Mots clés : Attachement; Personnalité; Violence conjugale psychologique}

La violence conjugale psychologique (VCP) est un ensemble de comportements problématiques envers son partenaire tels que les menaces au bien-être physique et la restriction de la liberté (Pico-Alfonso, 2005). II importe de se pencher sur la VCP perpétrée par des femmes, puisque celle-ci est sous-étudiée malgré son importante prévalence (Péloquin, Lafontaine \& Brassard, 2011).

Ce n'est que depuis récemment que les chercheurs montrent un intérêt grandissant envers des facteurs relationnels comme l'attachement pour expliquer la VCP. L'attachement correspond à la capacité de créer et de maintenir des liens avec des personnes significatives (Bowlby, 1973). À l'âge adulte, l'individu se situe sur deux continuums, appelés également les dimensions de l'attachement, soit l'anxiété d'abandon, qui correspond à la peur d'être rejeté, et l'évitement de l'intimité, qui réfère à de l'inconfort en situation de proximité (Brennan, Clark \& Shaver, 1998). En ce qui concerne les études liant l'attachement et la VCP, les résultats sont incohérents (Capaldi, Knoble, Shortt \& Kim, 2012), ce qui peut témoigner de l'existence de variables qui médient le lien entre les dimensions de l'attachement et la VCP (Péloquin et coll., 2011).

Parmi les potentielles variables médiatrices, il apparaît pertinent de s'intéresser à la personnalité puisqu'elle est liée à la façon dont l'individu entre en relation avec les autres et réagit à certaines situations (Costa \& McCrae, 1992). Le modèle à cinq facteurs de Goldberg (1981) surnommé «Big-Five» subdivise la personnalité en cinq traits, soit le névrosisme, l'agréabilité, l'extraversion, le caractère consciencieux et l'ouverture à l'expérience. Le but de la présente étude est d'examiner le potentiel rôle médiateur des traits de la personnalité du Big-Five dans l'explication du lien entre les dimensions de l'attachement et la VCP.

\section{Méthode}

L'échantillon est constitué de femmes consultant au Service de consultation de l'École de psychologie de I'Université Laval et ayant rempli une batterie de questionnaires autorapportés à la suite de leur première rencontre d'éva- luation. Le Revised Conflict Tactic Scale (Straus, Hamby, McCoy \& Sugarman, 1996) évalue la VCP, le questionnaire Experiences in Close Relationships scale (Brennan et coll., 1998) mesure les deux dimensions de l'attachement, et le NEO-Five Factor Inventory (Costa \& McCrae, 1992) a pour but de placer les individus sur les cinq traits de la personnalité du Big-Five. Une analyse acheminatoire est effectuée à l'aide de la version 7.3 du logiciel Mplus. Celle-ci permet de tester les effets indirects de l'attachement sur la VCP via la personnalité grâce à l'estimation simultanée d'une série de régressions. Les variables indépendantes sont l'anxiété d'abandon et l'évitement de l'intimité, la variable dépendante est la VCP et les variables médiatrices sont les cinq traits de la personnalité.

\section{Résultats et discussion}

Le modèle comprenant tous les traits de la personnalité et les deux dimensions de l'attachement explique $12 \%$ de la variance de la VCP. Les coefficients standardisés entre chacune des variables de l'étude sont présentés à la Figure 1.

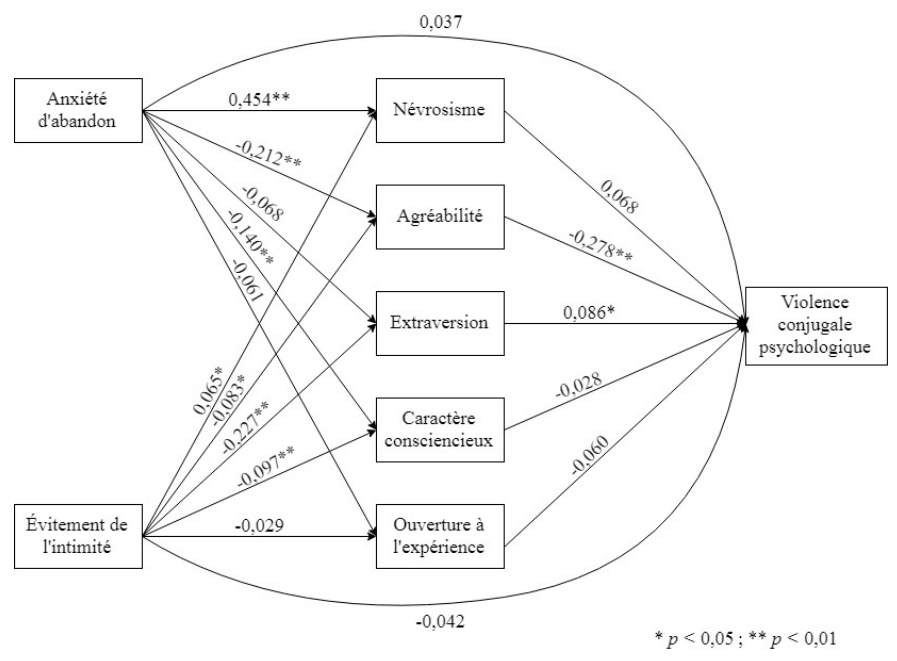

Figure 1. Coefficients de régression standardisés entre les deux dimensions de l'attachement, les cing traits de la personnalité et la violence conjugale psychologique.

L'agréabilité semble être un médiateur significatif de la relation entre les dimensions d'attachement et la VCP. D'une 
part, un haut niveau d'anxiété d'abandon serait lié à un bas niveau d'agréabilité, qui à son tour serait associé à un plus haut niveau de VCP. Il est supposé qu'une femme qui présente une plus grande peur d'être rejetée pourrait rester centrée sur cette détresse et ainsi utiliser la VCP comme une tentative mésadaptée pour conserver sa relation.

D'autre part, un haut niveau d'évitement de l'intimité serait lié à un bas niveau d'agréabilité qui à son tour serait associé à un haut niveau de VCP. Il est présumé qu'une femme qui repousse les relations intimes pourrait exprimer fortement son insatisfaction envers celles-ci et pourrait ainsi utiliser la VCP afin de repousser son partenaire et conserver une distance qui correspond à ses propres limites d'intimité.

De même, l'extraversion apparaît comme un médiateur significatif de l'association entre l'évitement de l'intimité et la VCP. Un bas niveau de l'évitement de l'intimité serait lié à un haut niveau d'extraversion qui à son tour serait associé à un plus haut niveau de VCP. Une femme qui n'est pas repoussée par les relations de proximité aurait tendance à aller vers les autres. Ainsi, si elle sent que sa liberté à avoir une vie sociale active dans son couple est brimée, elle pourrait utiliser la VCP pour s'affirmer dans ses insatisfactions.

Les résultats permettent de considérer la personnalité et I'attachement comme cibles d'intervention clinique. II serait toutefois intéressant pour les recherches futures d'explorer l'aspect dynamique de cette relation de façon temporelle et d'un point de vue dyadique afin d'avoir une compréhension plus complète de ces variables en lien avec la VCP.

\section{Références}

Bowlby, J. (1973). Attachment and Loss. Separation: anxiety and anger, Volume 2. New York, NY: Basic Books.

Brennan, K. A., Clark, C. L., \& Shaver, P. R. (1998). Self-report measurement of adult attachment: An integrative overview. Dans J. A. Simpson \& W. S. Rholes (Eds.), Attachment theory and close relationships (pp. 46-76). New York, NY: Guilford Press, 1998.

Capaldi, D. M., Knoble, N. B., Shortt, J. W., \& Kim, H. K. (2012). A systematic review of risk factors for intimate partner violence. Partner Abuse, 3(2), 231-280. doi:10.1891/19466560.3.2.231

Costa, P. T., \& McCrae, R. R. (1992). Revised NEO personality inventory and NEO Five Factor Inventory professional manual. Odessa, FL: Psychological Assessment Resources.

Goldberg, L. R. (1981). Language and individual differences: The search for universals in personality lexicons. Review of Personality and Social Psychology, 2(1), 141-165.

Péloquin, K., Lafontaine, M. F., \& Brassard, A. (2011). A dyadic approach to the study of romantic attachment, dyadic empathy, and psychological partner aggression. Journal of Social and Personal Relationships, 28(7), 915-942. doi:10.1177/0265407510397988

Pico-Alfonso, M. A. (2005). Psychological intimate partner violence: The major predictor of posttraumatic stress disorder in abused women. Neuroscience and Biobehavioral Reviews, 29, 181-193. doi:10.1016/j.neubiorev.2004.08.010

Straus, M. A., Hamby, S. L., Boney-McCoy, S., \& Sugarman, D. B. (1996). The revised conflict tactics scales (CTS2) development and preliminary psychometric data. Journal of Family Issues, 17(3), 283-316. doi:10.1177/019251396017003001

\section{Pour citer l'article}

Cloutier, G., Nolin, M.-C., Côté, L., Sabourin, S., \& Bellemare, V. (2019). Attachement, personnalité et violence conjugale psychologique chez des femmes en psychothérapie. Psycause: Revue scientifique étudiante de l'École de psychologie de I'Université Laval, 9(2), 5-6. 